\section{SCREENING FOR COELIAC DISEASE.}

T. Not,A.Ventura,M.Bittolo*,G. Torre and M.Andolina

Clinica Pediatrica and Dipart.di Biologia, Univers.Trieste;TALY Antfalfagliadin antibodies (AGA) are a useful tool in screening for
Coeliac Disease (CD). Anyway, this test requires a rather speciallzed laboratory, a venous bloog sample and about one week in order
to obtain the result for the famlylit paediatrician. We developed
and evaluated a dot immunobinding assay to detect alfagliadin IgG

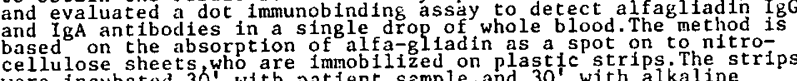

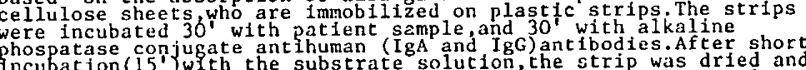
phospatase conjugate ant human (IgA and IgG) ant ibodies. Af ter short
incubation(15') wh the substrate solution, the strip was dried and
finally examined for the results, which are expressed by a colofinally examined for the results, which are expressed by a colo-
rimetric reaction. Twenty patients with cD(10 with active disease,
lo during gluten freediet (GFD)), w1 children with other gastro10 during gluten free diet (GFD)), 11 children with other gastro-
intestinal diseases ( 8 IBD, 1 autooimune chronic diarrhoea, 1 con-
genital microvillous atrophy, 1 unexplained chroufc diarrhoea) and genital microvillous atrophy, 1 unexplained chrouf diarrhoea) an
gh healty controls were examined with both strip AGA test and the
classic thisA test for AGA. The results are summarized as follows:

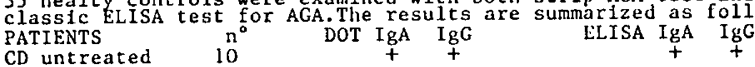
CD on GFD

GASTROINT.CONTR.

HEALTHY CONTROLS 35

The method showed good correspondence with ELISA AGA test: the proment. We think that this "strip AGA test" could be very convenien alth for screening and for the follow-up of CD. This test could
also be uselul in population and/or family screening for C.D.
LKM-1 ANTIBODY POSITIVE AUTOIMMUNE HEPATITIS IN 44 ANTIGEN CYTOCHROME P450 dDI AND IN VIVO PHENOTYPE S. Koletzko, H. Löhr, M. Eichelbaum, F. Borchard, C. Rittner, K. -H. Meyer zum Büschenfelde, M. Manns Kinderklinik \& Patholog. Institut, Univ. D-4000 Düsseldorf, and
I. Medizinische Klinik \& Rechtsmedizin, Univ. D-6500 Mainz, FRG.

A subgroup of autoimmune type chronic active hepatitis (AI-CAH) is associated with LKM-1 autoantibodies. They are directed against Cytochrome P450 db1 and inhibit its function in vitro. We observed LKM-1 positive AI-CAH and colitis in a 13 year old girl. Liver disease responded well to corticosteroids. Father, mother, brother, the patient and her identical twin sister were investigated for HLA class I-III phenotypes, auto-antibodies, in investigated for HLA class $\mathrm{I}-\mathrm{III}$ phenotypes, auto-antibodies, in
vitro inhibition of $\mathrm{P450} \mathrm{db} 1$, and in vivo phenotype for drug metabolism (sparteine) mediated by this enzyme. Both twins had the autoimmune HLA haplotype $A 1, B 8, D R 3, C 4 A-00$. While the mother is a homozygous extensive metabolizer (EM) (metabolic ratio 0.33), the father is a homozygous poor metabolizer (MR 65.25) and all children are heterozygote EM (MR 1.01, 0.99 \& 1.76). Only the index patient had signs of liver disease and was positive for LKM-1 antibodies, and only db1 catalysed oxidation of sparteine in vitro up to $90 \frac{6}{6}$; Conclusion: We describe for the first time occurence of LKM-1 for the disease. Since both twins are of the EM metabolizer type, i.e. express functionally intact P450 dbl, and share the i.e. express functionally intact $P 450 \mathrm{dbl}$, and share the autoimmune HLA haplotype, we conclude that environmental
trigger the manifestation of this autoimmune liver disease.
42 DISEASE BEFORE AND AFTER URSODEOXYOHOLIC ACID (UDCA) THERAPY.

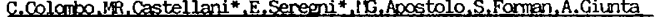
Dept.Pediatrics, Lniversity of Hilan, *Nuclear Medicine Div.National CancerInst. ITALY

It has recently been indicated that $96 \%$ of patients with liver disease secondary to Cystic Fibrosis ( $G$ ) showed evidence of common bile duct stenosis on hepatobiliary scanning (Gaskin et Al:N.Eng.J.Med.318:340,1988), we performed IDA scan pre and 10 12 months post adninistration of the hydrophilic and choleretic UDCA (15mg/Kg/day) in 8 childnen with $G$ related liver disease.Marphological pattern as well as functional parameters $\left(T^{2} / 2\right.$ of hepatic wash-out, time of visualizationof the intestine) were evaluated.At baseline, evidence of severe biliary obstruction was found in 2 cases, striking visualization of secondary and tertiary bile ducts in $\mathbf{5}$ and dilatation of cormon bile duct in 4 ; an enlanged gallbladder with delayed emptying was present in 2 patients and in 3 the gallbladder could not be visualized. After UDCA therapy, mor phologic appearance at IDA scan inanoved markedly in all patients: none showed evidence of biliary obstruction, dilatation of cormon bile duct and intrablepatic ducts was substantially reduced and in all cases the gallbladder was visualized and appea red to empty normally. Mean $1 / 2$ of hepatic wash-out decreased from $50+21$ min.at baseline to $36.0+18$ min.after therapy and mean time of visualization of the intesti ne decreased from $46.8+40.8 \mathrm{~min}$.to $17.5+9.3 \mathrm{~min}$.after UDCA. In all patients on UDCA liver function tests improved significantly and enrichment of the biliary bile acid pool with UDCA (from $4.8+2.2 \%$ to $28.5+9.6 \%$ ) occurred.

The modifications observed after UDCA therapy suggest that inspissated secretions in $C F$ patients may be responsible for the typical appearance at hepatobiliary scanning and that the inprovement during UDCA may be related to its choleretic effect.
PROSTAGLARDINS IN SMALL INTESIIMAL gUCOSA OF CHILDREN WITH ACTIYE COELLAC DISELSE AND OK A GLUTEM FREE DIET.

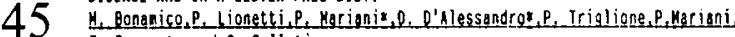
Ferrante and $G$ Ballati

I Paediatric Clinic, II Surgical Clinica, "La Sapienza' University, Rone, Italy. Enhanced synthesis of Prostaglandins (PGS) has been reported in coeliac disease (CD) and possible involvent of these substances in the pathogenesis of diarrhoea in coeliac patients has been supposed. The ail of our study was to evaluate Prostaglandin E2 (PGE2)

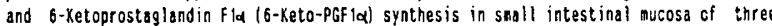
groups of patients: group $A$, consisting of 11 children with active $C D$ and total or subtotal nucossl atrophy group $B$ consisting of 7 children on a gluten free oiet for of lest 1 rear with nild villous atrohy or noral intestinal aucoss, group $C$ (controll of

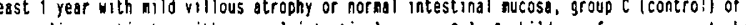

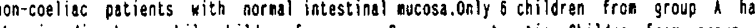
chronic diarthoed while children iron group b were asyaptonatic. Chiloren from group suffered from failure to thrive, ke excluded rroa this group children who shawed diarrhoea. The anounts of PGS generated by intestinal nucosa were messured by a standard nethod, using a RIA systen. In group A PGEZ generation was significantly higher 12052 401, Hean $\pm \mathrm{SE})$ than in group $C(603 \pm 140)(0<0.003)$. Children from group $A$ with chronic diarrhoes and those without this synpton both showed significant higker PSE2 generation $2569+687,1431+171$, respectively) than group $C(p(0.02)$. In group B PGE2 generation was higher $(1632+567)$ but not significantly different fron group C. E-Keto-PGFla generation although higher in group $A$ and $B$ than $C$ did not show any statistically significant variation. Our results indicate that PGE2 generation in CD is nct alvays related to the presence of diarrhoes. Elevated PGs levels in intestinal mucosa in CD may be due to both eahanced synthesis and decreased degradstion. Alternativeiy, hypothesized by Branski (J Pediats Gestroenteral Nutr 3,672, 1984), it aculd te the heothesize of on high PGE? levels in asyntonstic coeliac children on gluten free diet. "Waybe is recessary a long period of diet before to cease this adaptative nechanisn.
DO TRANS FATTY ACIOS (TFA) IMPAIR BIOSYNTHESIS OF

\section{3} LONG-CHAIN POLYUNSATURATES (LCP) IN MAN?

\section{Berthold Koletzko}

Universitäts-Kinderklinik D-4000 Düsseldorf, FRG.

Humans consume large amounts of TFA due to the extensive use of hydrogenated fats in food production. TFA consumption is considered safe for man, but side effects including impaired synthesis of LCP $(20 \& 22$ carbons) have been observed in animal studies. We have previously documented materno-fetal transfer of TFA in humans. Therefore, we looked for possible effects of TFA on LCP status during early life, when LCP modulate tissue growth and development. Methods: Blood plasma samples were obtained in 29 clinically well premature infants (gest. age $34.0+1.8$ wks., birthwt. $1694 \pm 173 \mathrm{~g}, M+S D$ ) prior to feeding in the morning of day birthwt. $1694 \pm 173 \mathrm{~g}, \mathrm{M}+\mathrm{SD}$ ) prior to feeding in the morning of day 3 post partum, when miTk intake was still very low. No infant had
received fat infusions. Fatty acids in lipid classes were received fat infusions. Fatty acids in lipid classes were Both total TFA and elaidic acid $(18: 1 \mathrm{t})$, the main dietary TFA were inversely correlated to LCP in plasma lipids. In triglycerides, linear correlation coefficients $(r)$ for 18:1t were significant (P<0.05* \& $\left.0.01^{\star *}\right)$ for $L C P\left(n-6-L C P:-0.41^{\star}, n-3-L C P\right.$ : $-0.50^{\star *}$, total LCP: $-0.55^{\star *}$ ) and for product substrate ratios of LCP biosynthes is $\left(20: 4 / 18: 2 n-6:-0.47^{\star \star}, 22: 6 / 18: 3 n-3:-0.50^{\star \star}\right)$. Similar results were found in other lipid classes for 18:1t and total TFA. Conclusions: 1. TFA exposure may impair biosynthes is of $n-6-$ and $n-3-L C P$ in man. 2 . Since the capacity for LCP of $n-6-$ and $n-3-L C P$ in man. 2 . Since the capacity for ICP biosynthesis is limited during early life and LCP accretion is
essential for normal functional development of membrane rich essential for normal functional development of membrane rich
tissues (e.g. brain), a high intrauterine TFA exposure may have serious risks for fetus and neonate. 3. This observation is the first indication of possible untoward effects of TFA in man.
SWLL. INTESTIMML TRNSPLNTATION IN (SIT) IN CHILPEN.

46 Goulet O.. Ravllion Y.. Jan D., Brousse N., Cart-Bensussan N. Mougenot J.F., De Potter S., Ricour C. Necker Hospital Paris.

After experiments on plglets and rats SIT using Cyclosporine A (CSA) and ofter obtaining consent form from parents, we performed six SIT in children (6mths9yrs) with short gut syndrom on home TPN for 0.5 to 6 years. All but one donor and reciplents were isoblood group $A B O$. with negotive cross motch reaction. Graft were hervested on braln dead neonates $(n=2)$ or children 6-17 yrs. $110 \pm 10 \mathrm{~cm}$ of Jejuno ileum underwent both vascular and luminal washing using collins $(n=3)$ or UW ( $n=3)$. After oorta and inferlor vena cava anastomosis total ischemic time ranged from $1 \mathrm{hr} 20$ to $6 \mathrm{hr} 30$. Groft was onestomosed on proximal and ; both distal graft and own intestine exteriorized os stomos. Initial Immunosuppression Included solumedrol $2 \mathrm{mg} / \mathrm{Kg} / \mathrm{d}$ and Cyclosporine as a continous intusion for RIA $\mathrm{m} / \mathrm{K} / \mathrm{d})$ for 14 days yere added in the last 4 coses. Systemic entibiotics and total decontemination of the graft are essociated. The first graft wos renoved after 8 hours for ischemia : the child was discherged on home PN. Eariy $1<15$ days) or deloyed (2-6 mths) acute graft rejection (GR) occured in all coses. Late clinical symptoms included increased lleostomy drainage while histologle pettern changed earlier including progressively : T cell infiltrates $(\mathrm{CO} 4+, \mathrm{CO} 8+, \mathrm{CO} 25+)$. increased HLA OR expression by crypts enterocytes, villi codeme, destruction of crypt and surface epithellum, crypt abcesse and finally mucosal sloughing. GR was treated with ALG or OKTS but leaded to graft removel in 3 cases 3 wks to 6 mths after SIT. One graft ras removed on the 17 th $\mathrm{mth}$ because of chronic rejection. Four Functionnol assesment included baryum tronsit, enzyme activities, and absorption tests. Those results show that SIT is possible but depending on important immunosuppression. No GVH reaction wos observed. GR con bo limited by repeated 